\title{
Evaluation of the relationship between neutrophil lymphocyte ratio and the most common bacterial urinary tract infections after transplantation
}

\author{
Kazimoglu $\mathrm{H}^{1}$, Uysal E², Dokur $\mathrm{M}^{3}$, Gunerkan $\mathrm{HR}^{4}$ \\ Sanko University School of Medicine, Department of Urology, Gaziantep, Turkey. hkazimoglu@hotmail.com
}

\begin{abstract}
OBJECTIVE: We aimed to evaluate the relationship between neutrophil lymphocyte ratio and the most common bacterial urinary tract infections after transplantation. We also assessed the frequency of bacterial infections, e.g. Escherichia coli, Klebsiella, and Enterococcus in the urinary tract, and determined the important factors affecting neutrophil/lymphocyte ratio (N/L).

METHODS: We compared the percentage of neutrophils (NE\%), percentage of lymphocytes (Lym\%), and N/L, along with blood urea nitrogen (BUN), creatinine, and white blood cells (WBC) in all patients before and after renal transplantation.

RESULTS: The Lym\% and WBC significantly increased after surgery while N/L\%, BUN, and creatinine levels were significantly decreased. Postoperative infections were evaluated by measuring WBC, NE\%, Lym\%, N/L\%, serum urea and creatinine levels, and no significant differences were seen compared to the preoperative values. Univariate analysis also did not show any significant differences between pre- and post-operative parameters. However, a significant difference in $\mathrm{N} / \mathrm{L} \%$ ratio was seen between the $E$. coli infected and uninfected recipients. CONCLUSIONS: Any significant difference in NE\%, Lym\%, and N/L\%, BUN, creatinine, and WBC parameters among infected and non-infected renal transplant patients were not found. The steps should be taken to prevent pre-transplantation infection and patients should be continuously monitored for infections post-transplantation (Tab. 6, Fig. 1, Ref. 24). Text in PDF www.elis.sk.

KEY WORDS: renal transplantation, urinary tract infection, neutrophil lymphocyte ratio.
\end{abstract}

\section{Introduction}

The incidence of chronic kidney disease is on the rise and many people die every year due to chronic kidney failure. The most effective treatment for chronic renal failure is undoubtedly organ transplantation, with over 17,600 kidney transplants performed in the United States in 2013 alone (1). After renal transplantation, 75 $\%$ of the patients contracted a viral and bacterial infection within the first year. Bacterial infections of the urinary tract are a common complication of renal transplantation and are classified as infections due to technical and anatomical abnormalities, urinary tract infections (UTI), pyelonephritis, the infections of the mucocutaneous surface, and mycobacterial infections $(2,3)$. Since gram-negative bacteria are causative agents of the most of these

${ }^{1}$ Sanko University School of Medicine, Department of Urology, Gaziantep, Turkey, ${ }^{2}$ Sanko University School of Medicine, Department of General Surgery, Gaziantep, Turkey, ${ }^{3}$ Biruni University School of Medicine, Department of Emergency, Istanbul, Turkey, and ${ }^{4}$ Toros Public Hospital, Department of Infection Diseases, Mersin, Turkey

Address for correspondence: H. Kazimoglu, MD, Sanko University School of Medicine, Department of Urology, Incilipinar Mah. Ali Fuat Cebesoy Bulv. No: 45 27090, Sehitkamil - Gaziantep, Turkey.

Phone: +90.3422115000 , Fax: +90.3422115010 infections, trimethoprim, sulfamethoxazole, fluoroquinolone, and other antibiotics that are effective against gram-negative bacteria are often used for those complications.

UTIs are common in the first three months after renal transplantation and are often the result of infected donor kidneys. The most dangerous and life-threatening complications that can arise due to transplantation-related UTIs are massive infective vesicoureteral reflux, polycystic disease, infected stones, and papillary necrosis (4). Therefore, the donors should be evaluated for UTIs before the transplantation.

Renal transplantation surgery removes the need for dialysis and improves the quality of life. Recent developments in immunosuppressive and anti-microbial therapies and surgery techniques have made renal transplantation the most preferred and successful treatment in the patients with renal failure (5). Renal transplantation can be performed using living as well as cadaveric organ donors. The survival rate of the transplant recipients from a living donor is about $97 \%$ within the first year and drops to $84 \%$ within five years. However, the survival rate in the recipients from cadaveric donors is $93 \%$ within the first year and drops to $75 \%$ within five years. The most dangerous complication post renal transplantation is UTI which develops at the frequency of $30 \%$ within the first six months $(6,7)$. 


\section{1-165}

Neutrophil-to-lymphocyte ratio (N/L) which was first described in 1967 as an indicator of ozone exposure, may be used as a marker of inflammation (8). NLR was shown as a beneficial inflammatory marker to assess outcome in surgery with cancer and cardiovascular diseases $(9,10)$. It may also be used to assess the outcome of kidney allograft function in renal transplant recipients (11). High NLR was inversely associated with good prognosis for patient and graft survival (11).

We aimed to evaluate the relationship between neutrophil lymphocyte ratio and the most common bacterial urinary tract infections after transplantation. We also assessed the frequency of bacterial infections, e.g. Escherichia coli, Klebsiella, and Enterococcus in the urinary tract, and determined the important factors affecting $\mathrm{N} / \mathrm{L}$.

\section{Materials and methods}

\section{Patient selection}

The present study was approved by Sanko University Medical Faculty Ethics Committee (2018/02/20). We included 213 patients (141 males, 73 females, and aged 5-65 years at the time of surgery), who had applied for renal transplantation to Private Sani Konukoglu Hospital (Sanko University, School of Medicine, Gaziantep, Turkey) for this study.

The patients were asked whether they want to participate in the study; after acceptance of patients, written informed consents were obtained from all participants. Patient details like demographic features, clinical/laboratory findings, comorbidities, intraoperative details and postoperative follow-up records were obtained from the file archives and hospital database. Only patients whose clinical records showed infection symptoms such as fever, malaise, uremia, dizziness and white ball height were selected and the patients were classified according to the presence or absence of UTIs.

\section{Patient monitoring}

The serum samples were analyzed for biochemical parameters, drug levels, and other hematological indices. In addition, the etiology of chronic renal failure, comorbidities, and immunosuppressive therapy were also examined. The recipients were followed up for $77.42 \pm 0.7$ months after transplantation to determine post-op infection or organ rejection.

\section{Biochemical parameters}

Neutrophil percentile (NE\%), lymphocyte percentile (Lym\%), neutrophil/lymphocyte $\%$ ratio (N/L\%), serum blood urea nitrogen (BUN) and creatinine and white blood cells (WBC) were evaluated in all patients before and after surgery. Preoperative Neutrophil and lymphocyte percentile were obtained from samples collected in the ethylenediaminetetraacetic acid tubes. Measurements of Neutrophil and lymphocyte were performed with a calibrated automatic hematology analyzer (Cell-dyn ruby,Abbott,Chicago,USA)

\section{Statistical analysis}

Normal distribution was checked by Shapiro-Wilk test. MannWhitney U-test was used to compare numerical variables between

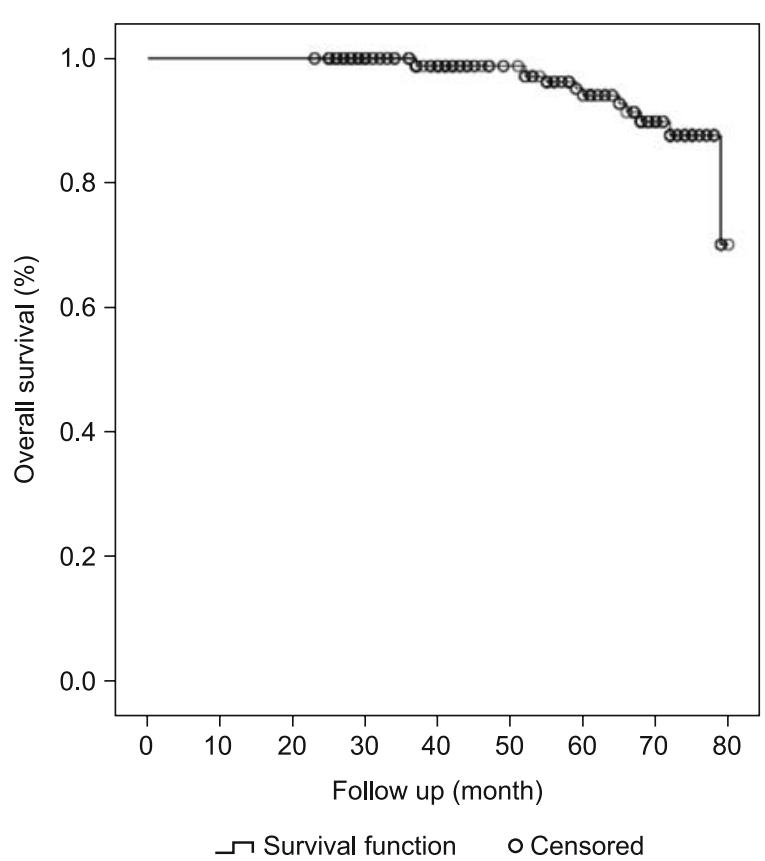

Fig. 1. Kaplan Meier curve for overall survival of patients.

groups. Generalized linear regression analyses were performed to determine important factors affecting N/L\%. Mean survival time was estimated by using Kaplan Meier method. All analyses were performed in SPSS for Windows version 24.0. A two-sided $p$ value $<0.05$ was considered statistically significant.

\section{Results}

The overall survival rates of all recipients were $100 \%$ in the first three years after transplantation and declined to $94.1 \%$ in the next two years (total five years). Mean survival duration was 77.42 \pm 0.7 months, during which 12 patients died (Fig. 1).

Biochemical analysis of serum indicated that Lym $\%$ and WBC levels significantly increased after surgery but the post-operative $\mathrm{N} / \mathrm{L} \%, \mathrm{BUN}$, and creatinine values were significantly decreased (Tab. 1). To determine whether in the patients who were infected postoperatively, $\mathrm{WBC}, \mathrm{NE} \%$, Lym $\%, \mathrm{~N} / \mathrm{L} \%$, urea, and creatinine

Tab. 1. Comparison of the biochemical parameters before and after surgery.

\begin{tabular}{lccccc}
\hline Variables & $\mathrm{n}$ & $\begin{array}{c}\text { Before } \\
\text { Surgery }\end{array}$ & $\mathrm{n}$ & $\begin{array}{c}\text { After } \\
\text { Surgery }\end{array}$ & $\mathrm{p}$ \\
\hline NE\% & 213 & $64.71 \pm 16.25$ & 213 & $64.1 \pm 12.45$ & 0.983 \\
Lym\% & 212 & $21.12 \pm 11.57$ & 212 & $23.43 \pm 9.38$ & $0.003^{*}$ \\
N/L\% & 209 & $16.31 \pm 41.99$ & 209 & $4.27 \pm 6.49$ & $0.001^{*}$ \\
BUN (mg/dL) & 213 & $45.71 \pm 20.87$ & 213 & $21.97 \pm 15.22$ & $0.001^{*}$ \\
Creatinine (mg/dL) & 213 & $7.72 \pm 2.86$ & 213 & $1.69 \pm 1.73$ & $0.001^{*}$ \\
WBC $\left(x 10^{3}\right)$ & 213 & $8.28 \pm 3.08$ & 213 & $9.23 \pm 7.77$ & $0.005^{*}$ \\
\hline
\end{tabular}

NE\%: percentage of neutrophils; Lym\%: percentage of lymphocyte; N/L\%: neutrophil/lymphocyte ratio; BUN (unit): serum blood urea nitrogen (mg/dL); WBC (unit): white blood cells (cells $/ \mathrm{mm} 3$ ); Creatinine (unit): serum creatinine $(\mathrm{mg} / \mathrm{dL}$ ) * Significant $<0.05$ level 
Tab. 2. Comparison of numerical measurements in the infected and non-infected groups.

\begin{tabular}{lccc}
\hline \multirow{2}{*}{ Variables } & \multicolumn{2}{c}{ Group } & \multirow{2}{*}{$\mathrm{p}$} \\
\cline { 2 - 3 } & Infection $(\mathrm{n}=92)$ & No infection $(\mathrm{n}=121)$ & \\
\hline WBC pre & $8.41 \pm 3.3$ & $8.19 \pm 2.91$ & 0.638 \\
WBC post & $9.75 \pm 11.16$ & $8.83 \pm 3.45$ & 0.729 \\
NE\% pre & $65 \pm 16.66$ & $64.49 \pm 16$ & 0.905 \\
NE\% post & $64.28 \pm 10.85$ & $63.96 \pm 13.57$ & 0.955 \\
LYM\% pre & $21.06 \pm 12.05$ & $21.17 \pm 11.23$ & 0.937 \\
LYM\% post & $23.73 \pm 8.28$ & $23.02 \pm 10.35$ & 0.566 \\
N/L\% pre & $19.6 \pm 50.74$ & $13.55 \pm 33.31$ & 0.965 \\
N/L\% post & $3.99 \pm 6.51$ & $4.49 \pm 6.49$ & 0.783 \\
Urea (BUN) pre & $43.76 \pm 19.17$ & $47.2 \pm 22.04$ & 0.326 \\
Urea (BUN) post & $22.11 \pm 14.31$ & $21.87 \pm 15.94$ & 0.889 \\
Creatinine pre & $7.54 \pm 2.86$ & $7.87 \pm 2.87$ & 0.444 \\
Creatinine post & $1.91 \pm 2.13$ & $1.52 \pm 1.35$ & 0.338 \\
\hline
\end{tabular}

NE\%: percentage of neutrophils; Lym\%: percentage of lymphocyte; N/L\%: neutrophil/lymphocyte ratio; BUN (unit): serum blood urea nitrogen $(\mathrm{mg} / \mathrm{dL}) ; \mathrm{WBC}$ (unit): white blood cells (cells/mm3); Creatinine (unit): serum creatinine (mg/dL)

* Significant $<0.05$ level

Tab. 3. General linear model to estimate post op N/L \% ratio.

\begin{tabular}{lcccc}
\hline \multirow{2}{*}{ Variables } & \multirow{2}{*}{ Beta } & \multicolumn{2}{c}{$95 \%$ Confidence Interval } & \multirow{2}{*}{$\mathrm{p}$} \\
\cline { 3 - 4 } & & Lower & Upper & \\
\hline Woman \& Man & -2.608 & -4.232 & -0.984 & $0.002 *$ \\
NELYM pre & .025 & -0.002 & 0.052 & 0.070 \\
Age & .014 & -0.061 & 0.089 & 0.716 \\
Hospitalization & -.032 & -0.163 & 0.100 & 0.638 \\
Number ATG & .001 & -0.002 & 0.005 & 0.446 \\
Infection Yes/No & -2.267 & -3.875 & -0.659 & $0.006^{*}$ \\
E. coli + vs - & -2.644 & -4.557 & -0.731 & $0.007 *$ \\
Klebsiella + vs - & 1.053 & -1.746 & 3.852 & 0.461 \\
Enterococci+ vs - & -0.783 & -4.925 & 3.360 & 0.711 \\
\hline * Significant at 0.05 lever & & & &
\end{tabular}

* Significant at 0.05 level

Tab. 4. Comparison of the measurements in the presence or absence of $E$. coli infections.

\begin{tabular}{lccc}
\hline \multirow{2}{*}{ Variables } & \multicolumn{2}{c}{ E. coli } & \multirow{2}{*}{$\mathrm{p}$} \\
\cline { 2 - 3 } & Presence $(\mathrm{n}=66)$ & Absence $(\mathrm{n}=147)$ & \\
\hline WBC pre & $8.72 \pm 3.58$ & $8.09 \pm 2.82$ & 0.379 \\
WBC post & $10.3 \pm 13.05$ & $8.75 \pm 3.34$ & 0.823 \\
NE\% pre & $65.92 \pm 16.28$ & $64.17 \pm 16.26$ & 0.641 \\
NE\% post & $63.75 \pm 11.62$ & $64.26 \pm 12.83$ & 0.752 \\
LYM\% pre & $20.71 \pm 12.54$ & $21.31 \pm 11.14$ & 0.758 \\
LYM\%post & $23.96 \pm 8.5$ & $23.04 \pm 9.93$ & 0.430 \\
N/L\% pre & $22.31 \pm 57.05$ & $13.4 \pm 32.48$ & 0.680 \\
N/L\% post & $3.49 \pm 3.06$ & $4.63 \pm 7.52$ & 0.541 \\
Urea (BUN) pre & $43.89 \pm 19.86$ & $46.53 \pm 21.32$ & 0.432 \\
Urea (BUN) post & $22.96 \pm 15.82$ & $21.53 \pm 14.98$ & 0.838 \\
Creatinine pre & $7.36 \pm 2.87$ & $7.89 \pm 2.86$ & 0.239 \\
Creatinine post & $1.97 \pm 2.25$ & $1.56 \pm 1.44$ & 0.319 \\
\hline
\end{tabular}

NE\%: percentage of neutrophils; Lym\%: percentage of lymphocyte; N/L\%: neutrophil/lymphocyte ratio; BUN (unit): serum blood urea nitrogen $(\mathrm{mg} / \mathrm{dL})$; WBC (unit): white blood cells (cells/mm3); Creatinine (unit): serum creatinine (mg/dL) * Significant $<0.05$ level

values were evaluated but no significant differences were seen relative to the preoperative values (Tab. 2).

Univariate analysis also did not indicate any significant difference between the parameters (Tab. 3). After adjusting for gender, age, hospitalization, number of ATG and pre-operative N/L\%, a significant difference was seen between the pre- and postopera-
Tab. 5. Comparison of the measurements in the presence or absence of Klebsiella infectious.

\begin{tabular}{lccc}
\hline \multirow{2}{*}{ Variables } & \multicolumn{2}{c}{ Klebsiella } & \multirow{2}{*}{$\mathrm{p}$} \\
\cline { 2 - 3 } & Presence $(\mathrm{n}=18)$ & Absence $(\mathrm{n}=195)$ & \\
\hline WBC pre & $9.03 \pm 3.58$ & $8.22 \pm 3.03$ & 0.272 \\
WBC post & $8.7 \pm 3.49$ & $9.28 \pm 8.06$ & 0.524 \\
NE\% pre & $66.98 \pm 14.71$ & $64.5 \pm 16.4$ & 0.542 \\
NE\% post & $65.93 \pm 9.68$ & $63.93 \pm 12.68$ & 0.795 \\
LYM\% pre & $23.17 \pm 10.92$ & $20.93 \pm 11.63$ & 0.382 \\
LYM\%post & $23.59 \pm 8.94$ & $23.3 \pm 9.57$ & 0.957 \\
N/L\% pre & $10.83 \pm 25.95$ & $16.69 \pm 43.01$ & 0.651 \\
N/L\% post & $3.76 \pm 3.08$ & $4.32 \pm 6.72$ & 0.896 \\
Ure (BUN) pre & $47.67 \pm 18.41$ & $45.53 \pm 21.12$ & 0.478 \\
Ure (BUN) post & $21.56 \pm 13.06$ & $22.01 \pm 15.44$ & 1.000 \\
Creatinine pre & $8.22 \pm 2.96$ & $7.68 \pm 2.86$ & 0.502 \\
Creatinine post & $1.73 \pm 1.6$ & $1.69 \pm 1.75$ & 0.727 \\
\hline
\end{tabular}

$\mathrm{NE} \%$ : percentage of neutrophils; Lym\%: percentage of lymphocyte; N/L\%: neutrophil/lymphocyte ratio; BUN (unit): serum blood urea nitrogen $(\mathrm{mg} / \mathrm{dL})$; WBC (unit): white blood cells (cells/mm3); Creatinine (unit): serum creatinine (mg/dL)

* Significant $<0.05$ level

Tab. 6. Comparison of the measurements according to the presence or absence of Enterococcus infectious.

\begin{tabular}{lccc}
\hline \multirow{2}{*}{ Variables } & \multicolumn{2}{c}{ Enterococcus } & \multirow{2}{*}{$\mathrm{p}$} \\
\cline { 2 - 3 } & Presence $(\mathrm{n}=18)$ & Absence $(\mathrm{n}=195)$ & \\
\hline WBC pre & $7.66 \pm 3.02$ & $8.34 \pm 3.09$ & 0.508 \\
WBC post & $9.35 \pm 2.73$ & $9.22 \pm 8.08$ & 0.417 \\
NE\% pre & $63.09 \pm 15.43$ & $64.86 \pm 16.35$ & 0.643 \\
NE\% post & $64.83 \pm 7.35$ & $64.03 \pm 12.83$ & 0.769 \\
LYM\% pre & $21.65 \pm 12.33$ & $21.07 \pm 11.52$ & 0.891 \\
LYM\%post & $24.66 \pm 6.65$ & $23.2 \pm 9.72$ & 0.742 \\
N/L\% pre & $23.98 \pm 69.49$ & $15.46 \pm 38.45$ & 0.907 \\
N/L\% post & $2.89 \pm 1.08$ & $4.4 \pm 6.76$ & 0.993 \\
Urea (BUN) pre & $41.83 \pm 20.92$ & $46.07 \pm 20.88$ & 0.300 \\
Urea (BUN) post & $21.74 \pm 15.28$ & $22 \pm 15.26$ & 0.952 \\
Creatinine pre & $6.88 \pm 2.62$ & $7.8 \pm 2.88$ & 0.134 \\
Creatinine post & $1.91 \pm 2.11$ & $1.67 \pm 1.7$ & 0.664 \\
\hline
\end{tabular}

$\mathrm{NE} \%$ : percentage of neutrophils; Lym\%: percentage of lymphocyte; N/L\%: neutrophil/lymphocyte ratio; BUN (unit): serum blood urea nitrogen $(\mathrm{mg} / \mathrm{dL})$; WBC (unit): white blood cells (cells/mm3); Creatinine (unit): serum creatinine (mg/dL) * Significant $<0.05$ level

tive $\mathrm{N} / \mathrm{L} \%(\mathrm{Beta}=-2,267, \mathrm{p}=0.006)$. Furthermore, a significant difference in $\mathrm{N} / \mathrm{L} \%$ was found between the $E$. coli infected and uninfected patients (Tab. 3). Finally, WBC, NE\%, Lym\%, N/L\%, urea, and creatinine values were not significantly different between the patients infected with E. coli, Klebsiella and Enterococcus and the non- infected patients (Tabs 4-6).

\section{Discussion}

We found that the Lym\% and WBC significantly increased after renal transplantation surgery while N/L\%, serum BUN, and creatinine levels were significantly decreased. When we comparing the renal transplant recipients whether they had postoperative infection or not, we found no differences in terms of Lym $\%, \mathrm{WBC}$, $\mathrm{N} / \mathrm{L}$, serum creatinine and BUN levels. Also; we did not find any differences among different microorganism's groups in terms of $\mathrm{Lym} \%, \mathrm{WBC}, \mathrm{N} / \mathrm{L}$, serum creatinine and BUN levels.

UTIs occur most frequently within the first six months posttransplantation (12-13). Valeria et al reported that $50 \%$ of all 


\section{1-165}

infections in the renal transplant recipients occurred within the first 44 days.(12) According to a retrospective study in 28,942 patients whose data were obtained from the Kidney Disease Data Bank in the United States, UTI was more common within the first six months (13). Takai et al reported the highest incidence of infections within the first year post-transplantation in a cohort of 363 kidney recipients (14). Most studies that investigated posttransplantation infections evaluated biochemical parameters like WBC, NE\%, LYM\%, N/L\%, urea, and creatinine. We did not observe any differences in these parameters between the patients who were infected with E. coli, Klebsiella, and Enterococcus and the non- infected patients [Table 4 (E. coli), Table 5 (Klebsiella), Table 6 (Enterococcus)]. We may speculate that biochemical screening for UTI in the transplant recipients should be verified by further clinical tests. However; we must keep in mind that the only clinical examination may also not be sufficient since asymptomatic bacteriuria is a common complication after renal transplantation (15).

Abbott et al found significant differences in UTIs between genders within the first six months, and the infection rates increased to $60 \%$ in women and $47 \%$ in men within three years' post-transplantation, indicating that gender is a risk factor for UTI (13). Takai et al also reported a higher infection rate in women (49 $\%$ ) compared to men (14\%) (14). Another study followed-up 500 kidney recipients for 42 months and found that the rates of UTI were $68 \%$ in women and $30 \%$ in men (16). In the present study, the infection rate was not significantly different between genders. There were no significant differences among genders in terms of the biochemical indices of infection, except for $\mathrm{N} / \mathrm{L} \%$.

$\mathrm{N} / \mathrm{L} \%$, a surrogate marker of inflammation, has been recently adopted to predict postoperative outcomes $(17,18)$. It also predicts the severity of clinical course in patients with chronic disease and in patients who had surgery (19). In the present study, we found that N/L\% did not show any significant differences between infected and noninfected patients.

UTI is generally caused by Escherichia coli (30-80\%) or other Gram-negative bacteria such as Klebsiella $(\approx 10 \%)$, Proteus $(\approx 5$ $\%$ ) or Pseudomonas aeruginosa $(\approx 10 \%)$. Gram-positive enterococci $(15-30 \%)$ or Staphylococcus aureus $(\approx 10 \%)$ is also found more often in UTI patients compared to the normal population (20-22). Our N/L ratio findings for E. coli infections is consistent with previous reports but that for Klebsiella and Enterococcus contradicts published reports $(\mathrm{p}<0.05)$ (Tab. 3). The transplant recipients have a $90 \%$ rate of UTI, and the frequencies of $E$. coli, Klebsiella, Enterococcus, Enterobacter, Pseudomonas aeruginosa, Citrobacter, A. baumannii, Staphylococcus and S. marcescensare reportedly $59.1 \%, 16.9 \%, 6.5 \%, 6.5 \%, 4 \%, 0.8 \%, 0.8 \%, 1.6$ $\%$ and $0.8 \%$, respectively (23). In another study evaluating 500 patients, the frequencies of E. coli, Enterococci, Staphylococci, and Klebsiella pneumoniae were $29 \%, 24 \%, 12 \%$, and $10 \%$, respectively (24).

Chronic renal failure, chronic pyelonephritis and diabetes mellitus and delayed postoperative graft function are the major risk factors for UTIs in patients with renal transplantation (23). The rate of UTI is $28 \%$ in the patients transplanted from cadaveric donors and $23 \%$ in those who are transplanted from living donors (16). Earlier studies indicate that acute UTIs affect the survival rate of the recipients but since the infected patients in our cohort were only positive for E. coli (Tab. 3), the overall survival rates of the patients were similar during the acute period and dropped to $0.6 \%$ at the end of 80 months (Fig. 1).

\section{Conclusion}

Although UTIs remain a significant cause of morbidity and mortality after renal transplantation, improved prophylactic, diagnostic, and treatment strategies might decrease the risk and negative effect of infection on transplantation outcomes. The steps should be taken to prevent pre-transplantation infection and patients should be continuously monitored for infections posttransplantation.

\section{References}

1. Kaplan JM, Sharma N, Dikdan S. Hypoxia-Inducible Factor and Its Role in the Management of Anemia in Chronic Kidney Disease. Int J Mol Sci 2018; 19 (2).

2. Rubin RH. Overview: pathogenesis of fungal infections in the organ transplant recipient.Transpl Infect Dis 2002; 4 (Suppl 3): 12-17.

3. Rubin RH, Ikonen T, Gummert JF, Morris RE. The therapeutic prescription for the organ transplant recipient: the linkage of immunosuppression and antimicrobial strategies. Transpl Infect Dis 1999;1: 29-39.

4. Coussement J, Scemla A, Abramowicz D, Nagler EV, Webster AC. Antibiotics for asymptomatic bacteriuria in kidney transplant recipients. Cochrane Database Syst Rev 2018; 2: CD011357.

5. Di Lullo L, House A, Gorini A, Santoboni A, Russo D, Ronco C. Chronic kidney disease and cardiovascular complications. Heart Fail Rev 2015; 20: 259-272.

6. Galindo Sacristan P, Perez Marfil A, Osorio Moratalla JM et al. Predictive factors of infection in the first year after kidney transplantation. Transplant Proc 2013; 45: 3620-3623.

7. Golebiewska J, Debska-Slizien A, Zadrozny D, Rutkowski B. Acute graft pyelonephritis during the first year after renal transplantation. Transplant Proc 2014; 46: 2743-2747.

8. Bobb GA, Fairchild EJ. Neutrophil-tolymphocyte ratio as indicator of ozone exposure. Toxicol Appl Pharmacol 1967; 11: 558-564.

9. Halazun KJ, Aldoori A, Malik HZ et al. Elevated preoperative neutrophil to lymphocyte ratio predicts survival following hepatic resection for colorectal liver metastases. Eur J Surg Oncol 2008; 34: 55e60.

10. Duffy BK, Gurm HS, Rajagopal V, Gupta R, Ellis SG, Bhatt DL. Usefulness of an elevated neutrophil to lymphocyte ratio in predicting long-term mortality after percutaneous intervention. Am J Cardiol 2006; 97: $993 \mathrm{e} 6$.

11. Halazun JL, Marangoni G, Hakeem A, Fraser SM, Farid SG, Ahmad N. Elevated pre-operative recipient neutrophil-lymphocyte ratio is associated with delayed graft function following kidney transplantation. Transplant Proc 2013; 45: 3245e57.

12. Valera B, Gentil MA, Cabello V, Fijo J, Cordero E, Cisneros JM. Epidemiology of urinary infections in renal transplant recipients. Transplant Proc 2006; 38: 2414-2415. 
13. Abbott KC, Swanson SJ, Richter ER et al. Late urinary tract infection after renal transplantation in the United States. Am J Kidney Dis 2004; 44: 353-362.

14. Takai K, Aoki A, Suga A et al. Urinary tract infections following renal transplantation.Transplant Proc 1998; 30: 3140-3141.

15. Yacoub R, Akl NK. Urinary tract infections and asymptomatic bacteriuria in renal transplant recipients. J Glob Infect Dis 2011; 3: 383-389.

16. Chuang P, Parikh CR, Langone A. Urinary tract infections after renal transplantation: a retrospective review at two US transplant centers. Clin Transplant 2005; 19: 230-235.

17. Malik HZ, Prasad KR, Halazun KJ et al. Preoperative prognostic score for predicting survival after hepatic resection for colorectal liver metastases. Ann Surg 2007; 246: 806-814.

18. Zahorec R. Ratio of neutrophil to lymphocyte counts - rapid and simple parameter of systemic inflammation and stress in critically ill. Bratisl Lek Listy 2001; 102: 5-14.
19. Rix TE, Bates T. Pre-operative risk scores for the prediction of outcome in elderly people who require emergency surgery. World J Emerg Surg 2007; 2: 16.

20. Alangaden GJ, Thyagarajan R, Gruber SA et al. Infectious complications after kidney transplantation: current epidemiology and associated risk factors. Clin Transplant 2006; 20: 401-409.

21. Saemann M, Horl WH. Urinary tract infection in renal transplant recipients. Eur J Clin Invest 2008; 38 (Suppl 2): 58-65.

22. Senger SS, Arslan H, Azap OK, Timurkaynak F, Cagir U, Haberal M. Urinary tract infections in renal transplant recipients. Transplant Proc 2007; 39: 1016-1017.

23. Memikoglu KO, Keven K, Sengul S, Soypacaci Z, Erturk S, Erbay B. Urinary tract infections following renal transplantation: a single-center experience. Transplant Proc 2007; 39: 3131-3134.

24. Dharnidharka VR, Agodoa LY, Abbott KC. Risk factors for hospitalization for bacterial or viral infection in renal transplant recipients - an analysis of USRDS data. Am J Transplant 2007; 7: 653-661.

Received October 21, 2018. Accepted November 28, 2018. 